

\title{
Remarks on modular symbols for Maass wave forms
}

\author{
Yuri I. Manin
}

To Professor F. Hirzebruch, with friendship and admiration, for his anniversary.

In this paper I introduce modular symbols for Maass wave cusp forms. They appear in the guise of finitely additive functions on the boolean algebra generated by intervals with nonpositive rational ends, with values in analytic functions (pseudomeasures in the sense of Manin and Marcolli). We explain the basic issues and draw an analogy with the $p$-adic case. We then construct the new modular symbols, followed by the related Lévy-Mellin transforms. This work builds on the fundamental study of Lewis and Zagier (2001).

\section{Introduction}

0.0. Summary. Maass wave cusp forms can be considered as analogs of classical cusp forms that have "complex weights" determined by the spectrum of the hyperbolic Laplace operator on the upper complex half-plane. In particular, Maass eigenforms with respect to all Hecke operators define interesting Dirichlet series, exactly as in the classical case.

Dirichlet series related to classical cusp forms admit $p$-adic analytic continuation. An efficient way to construct this continuation is based on the theory of modular symbols, which allows one to define first $p$-adic pseudomeasures and then integrate them in order to construct a $p$-adic version of the Mellin transform (the Mazur-Mellin transform; see [Mazur and Swinnerton-Dyer 1974; Višik and Manin 1974]).

In Section 1 we introduce modular symbols for Maass forms. We also transfer the construction of $p$-adic pseudomeasures back to the archimedean domain, and introduce the notion of $\infty$-adic integration and the respective Lévy-Mellin transform.

We argue that in the real analog of $\mathbb{Z}_{p}$ - the segment $[0,1]$ - the boolean algebra of closed/open $p$-adic subsets must be replaced by the boolean algebra of finite

Keywords: Maass modular forms, modular symbols. 
unions of intervals with rational ends, on which modular symbols, both classical and new, form a finitely additive pseudomeasure that can be used for Mellin-type integration. This is the theme of Section 2.

An important role in this theory is played by continued fractions and the dynamical system based on them. In the last subsections I show that the respective transfer operator can be treated as the "Hecke operator $T_{1}$ " corresponding to the "prime" in characteristic zero, and that the modified Brjuno function in the theory of linearization of holomorphic maps [Marmi et al. 2001; 2006] can be used for the calculation of derivatives of classical Dirichlet series related to cusp forms, replacing the eta function appearing in [Goldfeld 1995].

These ideas are explained in more detail below. In the proofs, I make heavy use of the fundamental study [Lewis and Zagier 2001].

0.1. Period polynomials and period functions. Let $u(\tau) \in S_{2 k}(\operatorname{SL}(2, \mathbb{Z}))$ be a cusp form of integer weight $2 k>0$ for the full modular group. This means that it is holomorphic in the upper half-plane, the tensor $u(\tau)(d \tau)^{k}$ is $\operatorname{SL}(2, \mathbb{Z})$-invariant, and $u(\tau)$ vanishes at cusps.

Its period polynomial is defined as the integral

$$
\psi(z)=\psi_{u}(z):=\int_{0}^{i \infty} u(\tau)(z-\tau)^{2 k-2} d \tau .
$$

Here $z$ is, for the time being, an auxiliary formal variable.

One remarkable discovery in the theory of modular functions was the possibility of developing versions for a certain set of complex weights $2 s$ (replacing the former $2-2 k$ ). This spectrum consists of the (doubled) zeroes of Selberg's zeta function $Z(s)$ of $\operatorname{SL}(2, \mathbb{Z})$ acting on the upper half-plane, or equivalently, those values of $s$ for which the Mayer transfer operator $\mathscr{L}_{s}^{2}$ [Mayer 1991a; 1991b] has 1 as its eigenvalue: see [Lewis and Zagier 1997] for a short review and [Lewis and Zagier 2001] for a comprehensive exposition.

0.2. Classical modular symbols. The classical modular symbols of weight $2 k$ for $\mathrm{SL}(2, \mathbb{Z})$, in one of their guises, can be defined simply as the integrals

$$
\int_{\alpha}^{\beta} u(\tau)(z-\tau)^{2 k-2} d \tau
$$

where this time $\alpha, \beta \in \mathbb{P}^{1}(\mathbb{Q})$ are arbitrary cusps, and the integration is taken along, say, the hyperbolic geodesic connecting $\beta$ to $\alpha$.

More precisely, the modular symbol $\{\alpha, \beta\}_{k}$ (for the full modular group) is the integral (0.2) considered as a linear map

$$
\{\alpha, \beta\}_{k}: S_{2 k}(\operatorname{SL}(2, \mathbb{Z})) \rightarrow \mathbb{C}[z]
$$


In the next subsections, we will briefly recall the number-theoretic motivations for considering (0.3). A geometric interpretation of (0.3), after a dualization, runs as follows: this integral expresses the pairing between the Hodge cohomology and the Betti homology of the moduli space $\bar{M}_{1,2 k-2}$ of elliptic curves with marked points. (See [Shokurov 1980a; 1980b] for a version involving Kuga varieties rather than moduli spaces.)

The modular symbols (0.3) satisfy the simple functional equations

$$
\{\alpha, \beta\}_{k}+\{\beta, \gamma\}_{k}+\{\gamma, \alpha\}_{k}=0, \quad\{\alpha, \beta\}_{k}+\{\beta, \alpha\}_{k}=\{\alpha, \alpha\}_{k}=0 .
$$

Thus they can be extended to a $\mathbb{C}[z]$-valued finitely additive function on the boolean algebra generated by (positively oriented) segments with rational ends in $\mathbb{P}^{1}(\mathbb{R})$. We sometimes call such a function a pseudomeasure, as in [Manin and Marcolli 2008]. The variable change formula applied to (0.2) leads to an additional property of this particular pseudomeasure, which we call its modularity:

$$
\{g(\alpha), g(\beta)\}_{k}=g\{\alpha, \beta\}_{k} .
$$

Here $g \in \mathrm{SL}(2, \mathbb{Z})$ acts on $\mathbb{P}^{1}(\mathbb{Q})$ by fractional linear transformations, and on polynomials of degree at most $2 k-2$ by a natural twisted action.

A pseudomeasure can in principle take values in any abelian group, and the modularity condition $(0.5)$ makes sense if this group is a left $\operatorname{SL}(2, \mathbb{Z})$-module. If the group of values has no 2- and 3-torsion, the last two equations in (0.4) follow from the first one.

0.3. Modular symbols for Maass cusp forms. Our first goal is to extend the definition of $\{\alpha, \beta\}_{k}$ to complex weights for which there exist nontrivial Maass cusp forms. We take the formula (0.2) as our starting point and look for its analogs in Lewis-Zagier theory. We are interested mostly in complex critical zeroes/weights for which $\operatorname{Re} s=\frac{1}{2}$.

Tracing parallels with the classical theory, one should keep in mind that certain classical objects have more (or less) than one parallel in the new setting.

For example, the most straightforward analogs of $u(\tau) \in S_{2 k}(\operatorname{SL}(2, \mathbb{Z}))$ are apparently Maass wave cusp forms [1949] - smooth SL(2, ZZZ)-invariant functions on $H$ satisfying the hyperbolic Laplace equation $\Delta u=s(1-s) u$ and certain growth and vanishing conditions. An appropriate version of the period polynomial (0.1) for such a form is its period function $\psi_{u}(z)$, this time a holomorphic function of our former auxiliary variable $z$.

However, the relationship between $u$ and $\psi_{u}$, as it is first explained in [Lewis and Zagier 2001, Chapter I, Section 1], does not look at all like (0.1) and passes through three intermediate steps: $u \leftrightarrow L_{\varepsilon} \leftrightarrow f \leftrightarrow \psi$. 
To the contrary, the structure of $(0.1)$ is reproduced in the formula

$$
\psi(z)=\int_{-\infty}^{0}(z-t)^{-2 s} U(t) d t
$$

(see [Lewis and Zagier 2001, p. 221]), in which $U(t) d t$ denotes a certain distribution on $\mathbb{R}$, called the boundary value of $u(\tau)$. Therefore, it is this distribution that in our context seems to be a more adequate analog of a classical cusp form, the more so that its $\mathrm{SL}(2, \mathbb{Z})$-invariance property involves an explicitly weighted action of the modular group,

$$
U\left(\frac{a t+b}{c t+d}\right)=|c t+d|^{2-2 s} U(t),
$$

whereas a Maass form is simply $\operatorname{SL}(2, \mathbb{Z})$-invariant.

Formula (0.6) seems to offer a straightforward way to define the modular symbol - just consider the integrals

$$
\int_{\alpha}^{\beta}(z-t)^{-2 s} U(t) d t .
$$

Formal manipulations with such integrals are simple and seemingly prove $(0.4)$ and (0.5); we reproduce them for their heuristic value. However, these calculations cannot be taken literally, because the characteristic functions of the intervals with rational ends do not belong to the space of test functions for the distribution.

Thus we have to find a way around this difficulty.

In fact, there are at least two different ways. One of them starts with the three-term functional equation for the period function $\psi(z)$, proceeds with pure algebra, and works also for Lewis-Zagier's "period-like functions".

Another method is applicable only to the period functions of Maass forms $u$ and uses the Lewis-Zagier formula of the form

$$
\psi(z)=\int_{-\infty}^{0}\left\{u, R_{z}^{s}\right\}(\tau)
$$

where the integrand is a closed 1-form depending on $z$ as a parameter (its structure is described in the main text below). One can then integrate this form along a path that may this time connect two arbitrary cusps, thus getting another analog of (0.2).

These two constructions form the content of Section 1.

0.4. The Mellin transform and classical modular symbols. Now we will explain some of our motivations.

Briefly, we want to describe a construction presenting the Maass Dirichlet series as an integral over, say, [0,1/2], formally similar to the Mazur-Mellin transform in the theory of $p$-adic interpolation. We call such a representation the $\infty$-adic 
Lévy-Mellin transform [Manin and Marcolli 2008]. The integration measure in both cases is constructed out of modular symbols.

Here is a sketch of the classical $p$-adic constructions. The classical theory of modular symbols, as presented in [Manin 1972; 1973], started with the following observations. Suppose that we are interested in the calculation of some values (say, at integer points $\rho$ ) of a Dirichlet series

$$
L_{\kappa}(\rho)=\sum_{n=1}^{\infty} a_{n} \kappa(n) n^{-\rho},
$$

where $\left(a_{n}\right)$ is a certain "arithmetic" function, and $\kappa$ is an additive character of $\mathbb{Z}$ of finite order. In the standard approach one first introduces the Fourier series

$$
u_{\kappa}(\tau):=\sum_{n=1}^{\infty} a_{n} \kappa(n) e^{2 \pi i n \tau}
$$

and then works with the Mellin transform

$$
\Lambda_{\kappa}(\rho):=\int_{0}^{i \infty} u_{\kappa}(\tau)\left(\frac{\tau}{i}\right)^{\rho-1} d \tau,
$$

which is related to $(0.8)$ by the simple formula $\Lambda(\rho)=i(2 \pi)^{-\rho} \Gamma(\rho) L(\rho)$.

Now, let $u(\tau):=u_{\kappa_{0}}(\tau)$ where $\kappa_{0}$ is identically 1. Clearly, $u_{\kappa}(\tau)=u(\tau+\alpha)$ for a rational number $\alpha$ such that $\kappa(n)=e^{2 \pi i \alpha n}$, so we can write, shifting the integration path,

$$
\Lambda_{\kappa}(\rho):=\int_{\alpha}^{i \infty} u(\tau)\left(\frac{\tau-\alpha}{i}\right)^{\rho-1} d \tau .
$$

Thus, if $\rho \geq 1$ is an integer, varying $\kappa$ in (0.8) reduces to replacing $\tau^{\rho-1}$ in $(0.10)$ by an arbitrary polynomial of degree $\leq \rho-1$ and allowing the integration paths $(\alpha, i \infty)$ with an arbitrary rational $\alpha$.

Furthermore, if $u \in S_{2 k}(\operatorname{SL}(2, \mathbb{Z}))$ as above, and $1 \leq \rho \leq 2 k-1$, applying to $\alpha$ the "continued fractions trick", we can replace $(\alpha, i \infty)$ by a sum of geodesic paths in the upper half-plane, joining pairwise cusps of the form $g^{-1}(0)$ and $g^{-1}(i \infty)$, where $g$ varies in $\operatorname{SL}(2, \mathbb{Z})$, and then return to $(0, i \infty)$ by transforming the integrand via $\tau \mapsto g \tau$. Thus, in particular, all values of (0.8) corresponding to integer $\rho$ inside the critical strip and arbitrary characters $\kappa$, can be expressed as linear combinations of modular symbols with rational coefficients, and span a finite-dimensional space over $\mathbb{Q}$.

0.5. The p-adic Mellin-Mazur transform. Such expressions were used in [Manin $1973 ; 1974]$ to produce a $p$-adic interpolation of values $(0.8)$. This problem will make sense if (after an appropriate normalization) these values lie in a finitely generated $\mathbb{Z}$-module, so the basic problem is to control the denominators. 
As we already said, the main tool for such an interpolation was a $p$-adic integral (the Mellin-Mazur transform) with respect to a $p$-adic pseudomeasure (see below) constructed using modular symbols. This transform integrates $\tau^{\rho-1}$ twisted by $\kappa$ against this pseudomeasure, and for finite order $\kappa$ produces the classical values $L_{\kappa}(\rho)$ more or less by definition. (In fact, one works usually with Dirichlet characters in place of $\kappa$, but the only difference consists in the appearance of auxiliary Gauss sums).

Here are some details.

(a) The p-adic integration domain and a naive pseudomeasure. The following tentative construction applies to any (absolutely convergent) series of the type (0.8) considered as a function of variable $\kappa$ with fixed $\rho$.

At the first approximation, consider $\mathbb{Z}_{p}$ with $\mathbb{Z}$ densely embedded in it. The boolean algebra of closed/open subsets of $\mathbb{Z}_{p}$ is generated by the primitive subsets $a+p^{m} \mathbb{Z}_{p}$, for $m=0,1,2, \ldots$ and $a$ ranging over all classes modulo $p^{m}$. Put

$$
\mu_{L}\left(a+p^{m} \mathbb{Z}_{p}\right):=\sum_{n \equiv a \bmod p^{m}} a_{n} n^{-\rho} .
$$

Any two primitive subsets either do not intersect, or one of them is contained in the other. If one primitive subset $I$ is a disjoint union of a finite family of other primitive subsets $I_{j}$, then $\mu_{L}(I)=\sum_{j} \mu_{L}\left(I_{j}\right)$. Thus $\mu_{L}$ extends to a $\mathbb{C}$-valued finitely additive function on the boolean algebra of closed/open subsets of $\mathbb{Z}_{p}$. We will call such objects pseudomeasures on $\mathbb{Z}_{p}$.

Generally, there is no chance that such a pseudomeasure will tend $p$-adically to zero when $m \rightarrow \infty$, even if its values lie in a finite-dimensional $\mathbb{Q}$-space. As explained in [Manin 1973], a Mazur's $p$-adic integral of a function against such a pseudomeasure typically converges not because the smaller primitive subsets have asymptotically vanishing pseudomeasure, but because in a typical Riemann sum, many approximately equal terms of not very large $p$-adic size are involved, and the quantity of summands $\approx p^{m}$, tending to zero $p$-adically, produces an unconventional nonarchimedean convergence effect.

If the pseudomeasure of small subsets does not tend to zero, the best one may hope for is that it will be bounded, i.e., its values will lie in a $\mathbb{Z}$-module of finite type. Even this usually will not happen: for example, one can suspect that

$$
\mu_{L}\left(p^{m} \mathbb{Z}\right)=\sum_{n \equiv 0 \bmod p^{m}} a_{n} n^{-\rho}=p^{-m \rho} \sum_{n} a_{n p^{m}} n^{-\rho}
$$

will have denominator of order $p^{-m \rho}$.

A radical way to avoid this danger is to postulate that $a_{n}=0$ if $n$ is divisible by $p$. One can achieve this cheaply, if $L$ admits an Euler product: simply discard the $p$-th Euler factor of $L$. 
(Notice an interesting archimedean analogy: the Mellin transform $\Lambda$ in $(0.10)$ produces $L$ supplemented by the initially missing "Euler factor at arithmetical infinity", where that phrase mean, as usual, the archimedean valuation of $\mathbb{Q}$.)

Returning to $L_{(p)}:=L$ divided by its $p$-factor, we may from now on look only at the group of $p$-adic units $\mathbb{Z}_{p}^{*} \subset \mathbb{Z}_{p}$ by which our pseudomeasure is now supported.

We repeat, in conclusion, that the classical values $(0.8)$ are tautologically integrals of the locally constant function $\kappa$ against our pseudomeasure $(0.12)$. (Of course, this is why chose it in the first place.) Only when we start to interpolate and allow, say, continuous $p$-adically valued multiplicative characters in place of $\kappa$ will we need the basics of such $p$-adic integration.

(b) Normalized p-adic pseudomeasure. Now let $L$ be the Mellin transform of an $\mathrm{SL}(2, \mathbb{Z})$-cusp form of weight $2 k$ as above. Representing the characteristic function of the set $a+p^{m} \mathbb{Z}$ by a linear combination of the additive characters $\kappa$ modulo $p^{m}$, and calculating $\Lambda_{\kappa}(\rho)$ as in $(0.4)$, we see that $\mu_{L}\left(a+p^{m} \mathbb{Z}_{p}\right)$ is a linear combination of modular symbols $\left\{b p^{-m}, i \infty\right\}, b \in \mathbb{Z}$.

Conversely, we may take an appropriate linear combination of such measures and obtain the one that was used in [Manin 1973; 1974], namely

$$
\mu_{p}\left(a+p^{m} \mathbb{Z}_{p}\right):=\varepsilon^{-m}\left\{a p^{-m}, i \infty\right\}_{k}-p^{2 k-2} \varepsilon^{-m+1}\left\{a p^{-m+1}, i \infty\right\}_{k} .
$$

Here $\varepsilon$ is a root of the (inverted) $p$-factor of $L: \varepsilon^{2}-a_{p} \varepsilon+p^{2 k-1}=0$. If one of the two roots is a $p$-adic unit, we get a bounded measure. In any case, its growth can be controlled. The appearance of two summands and $\varepsilon$ in (0.13) is a slightly more sophisticated solution than the total discarding of the $p$-th Euler factor.

0.6. The $\infty$-adic Lévy-Mellin transform. As suggested in [Manin and Marcolli 2008], we make the following replacements in the picture sketched above.

Replace $p$ by arithmetic infinity. Replace $\mathbb{Z}_{p}^{*}$ by the semiinterval $(0,1]$.

Call the classical Farey intervals with ends $\left(g^{-1}(i \infty), g^{-1}(0)\right), g \in \operatorname{SL}(2, \mathbb{Z})$, primitive segments. They will be our replacement for the residue classes $a+p^{m} \mathbb{Z}_{p}$. Exactly as residue classes, two open primitive segments either do not intersect, or one of them is contained in another. For an abelian group $W$, call a pseudomeasure a $W$-valued finitely additive function on segments with rational ends (see additional details below).

A typical pseudomeasure in this sense is the modular symbol itself:

$$
\mu(\alpha, \beta)=\{\alpha, \beta\}_{k} ;
$$

in particular, $\mu(\alpha, \infty)=\{\alpha, \infty\}_{k}$, which may be compared to (0.13).

As in the $p$-adic case, the pseudomeasure of a small segment is not small in the archimedean sense. However, now we cannot hope to compensate for this by the nonarchimedean effect referred to above. 
Instead, we suggest using the following general feature of our constructions:

The Mellin transform of a cusp form, after suitable normalization, can be naturally written as the sum over rational numbers in $(0,1]$ of values of a certain arithmetic function a:

$$
A:=\sum_{\beta \in(0,1] \cap \mathbb{Q}} a(\beta) .
$$

The values $a(p / q)$ involved here are essentially modular symbols divided by a power of the denominator $q$. For details, see Section 2 .

Generally, a convergent series of the form (0.14) gives rise to an archimedean integral in two related ways:

(i) The first construction. We can define a pseudomeasure $\mu=\mu_{a}$ on the boolean algebra generated by segments with irrational ends in $[0,1]$ putting

$$
\mu(\alpha, \beta):=\sum_{\gamma \in(\alpha, \beta) \cap \mathbb{Q}} a(\gamma)
$$

so that

$$
A=\int_{0}^{1} d \mu .
$$

One can also treat (0.14) as a distribution on an appropriate space of test functions.

This is a direct analog of $(0.12)$, however, it is not the version that we will use in this paper.

(ii) The second construction. Let $r$ be a function defined on pairs of positive coprime integers $(p, q), p<q$ and decreasing sufficiently fast. For a real number $\xi$, denote by $q_{i}(\xi)$ the denominator of the $i$-th convergent to $\xi, i \geq 0$. We can introduce the Lévy 1-form $l(\xi) d \xi$, associated to $r$ and defined on $\left(0, \frac{1}{2}\right]$ by the prescription

$$
l(\xi)=l_{r}(\xi)=\sum_{i=0}^{\infty} r\left(q_{i}(\xi), q_{i+1}(\xi)\right) .
$$

According to a lemma by P. Lévy, for any pair $(p, q)$ as above, the set of all $\xi \in\left(0, \frac{1}{2}\right]$ for which there exists $i$ with $(p, q)=\left(q_{i}(\xi), q_{i+1}(\xi)\right)$, fills a primitive semiinterval of length $1 /((p+q) q)$. Moreover, this $i$ is uniquely defined. Therefore, when $r(p, q)$ decreases sufficiently rapidly to assure convergence, we get

$$
\int_{0}^{1 / 2} l_{r}(\xi) d \xi=\sum_{\alpha=p / q \in(0,1]} \frac{r(p, q)}{(p+q) q} .
$$

In particular, we get $A$ from (0.14) if we choose

$$
r(p, q):=a(p / q)(p+q) q .
$$


When $A$ comes from a modular form (classical or Maass), so that the summands $a(\beta)$ are concocted of (classical or Maass) modular symbols, we will call the integral in (0.12) the $\infty$-adic Lévy-Mellin transform.

The Lévy functions and their generalizations appear also in a different context: that of linearizations of the germs of analytic diffeomorphisms of one complex variable $z$ with an indifferent fixed point. For example, a germ with linear part $e^{2 \pi i \xi} z$ is linearizable if and only if the Brjuno number of $\xi$,

$$
b(\xi):=\sum_{i=0}^{\infty} \frac{\log q_{n+1}(\xi)}{q_{n}(\xi)},
$$

is finite. In fact, an interesting theory is developed/reviewed in [Marmi et al. 2001; 2006] for another Brjuno function $B(\xi)$, which differs from $b(\xi)$ by $O(1)$, but satisfies a functional equation and has a complex version closely resembling some constructions in the theory of modular forms. In our context, it can be used for calculation of the derivative of some classical $L$-series at certain points. This looks like an interesting variation on the subject of the Lévy-Mellin transform.

0.7. A summary of p-adic/ $\infty$-adic analogies. For clarity, we summarize the suggested analogies as follows:

$$
\begin{array}{rll}
\mathbb{Z}_{p}^{*} & \Longleftrightarrow & (0,1] \\
\cup & \cup & \mathbb{Q} \cap(0,1] \\
\mathbb{Z} & \Longleftrightarrow & \text { primitive (Farey) segments } \\
a+p^{m} \mathbb{Z}_{p} & \Longleftrightarrow & \sum_{0<p / q \leq 1} \frac{a(p / q)}{q^{\rho}} \\
\sum_{m=1}^{\infty} \frac{a_{m}}{m^{\rho}} & \Longleftrightarrow &
\end{array}
$$

Mazur-Mellin transform $\Longleftrightarrow$ Lévy-Mellin transform

\section{Pseudomeasures associated with period-like functions}

1.1. A heuristic construction. For the moment, we adopt the viewpoint of [Lewis and Zagier 2001, Chapter II, Section 5]. Fix a complex number $s$ such that $s(1-s)$ is an eigenvalue of the standard hyperbolic Laplace operator on $\mathbb{C}$ producing a $\operatorname{PSL}(2, \mathbb{Z})$-invariant Maass wave form $u(z)=u_{s}(z), z \in H$. Define complex powers by the usual formula $t^{s}:=e^{s \log t}$ where the branch of the logarithm is determined by the normalization $-\pi<\arg t \leq \pi$. As shown in [Lewis and Zagier 1997], there exists a distribution $U(t)=U_{s}(t)$ on $\mathbb{R}$ whose values on the test functions of $t$ given by

$$
(\operatorname{Im} z)^{s}|z-t|^{-2 s}, \quad(z-t)^{-2 s}, \quad \chi_{(-\infty, 0)}(t)(z-t)^{-2 s}
$$


(where $z$ enters as a parameter) are respectively $u(z)$ (the initial Maass form), a function $f(z)$ holomorphic in $\mathbb{C} \backslash \mathbb{R}$, and a period function $\psi(z)$ defined and holomorphic in $\mathbb{C}^{\prime}:=\mathbb{C} \backslash(-\infty, 0]$. Here $\chi$ is the characteristic function of $\mathbb{R}_{-}$; in other words,

$$
\psi(z)=\int_{-\infty}^{0}(z-t)^{-2 s} U(t) d t .
$$

The distribution $U$ is automorphic in the following sense: for all

$$
g=\left(\begin{array}{ll}
a & b \\
c & d
\end{array}\right) \in \operatorname{SL}(2, \mathbb{Z})
$$

we have

$$
U\left(\frac{a t+b}{c t+d}\right)=|c t+d|^{2-2 s} U(t) .
$$

Thus, (1.1) has the same structure as (0.1).

Now consider only $g \in \operatorname{SL}(2, \mathbb{Z})$ with nonnegative entries. Then for any $z \in \mathbb{C}^{\prime}$ we have also $g z:=(a z+b) /(c z+d) \in \mathbb{C}^{\prime}$. From (1.2) we find formally

$$
\psi(g z)=\int_{-\infty}^{0}(g z-t)^{-2 s} U(t) d t=\int_{g^{-1}(-\infty)}^{g^{-1}(0)}(g z-g \tau)^{-2 s} U(g \tau) d(g \tau) .
$$

A direct calculation using (1.2) reduces the integrand to the form

$$
\left[\frac{z-\tau}{(c z+d)(c \tau+d)}\right]^{-2 s}|c \tau+d|^{-2 s+2} U(\tau) \frac{d \tau}{|c \tau+d|^{2}} .
$$

Since $a \neq 0$, we have

$$
g^{-1}(-\infty)=-\frac{d}{c}<-\frac{b}{a}=g^{-1}(0),
$$

and hence for $\tau \in\left(g^{-1}(-\infty), g^{-1}(0)\right)$ we have $c \tau+d>0$. This shows that all terms involving $c \tau+d$ in (1.4) cancel, so that finally we find formally

$$
\psi(g z)=(c z+d)^{2 s} \int_{-d / c}^{-b / a}(z-\tau)^{-2 s} U(\tau) d \tau .
$$

Thus if $(\alpha, \beta)=\left(g^{-1}(-\infty), g^{-1}(0)\right)$ with $g$ as above, and if we put

$$
\mu(\alpha, \beta)(z):=(c z+d)^{-2 s} \psi(g z)=\int_{\alpha}^{\beta}(z-t)^{-2 s} U(t) d t,
$$

then for three intervals $(\alpha, \beta),(\beta, \gamma),(\alpha, \gamma)$ of this type, we would have from (1.6)

$$
\mu(\alpha, \beta)(z)+\mu(\beta, \gamma)(z)=\mu(\alpha, \gamma)(z) .
$$

As we will see, all primitive intervals in $\mathbb{R}_{-}$are of this form, so we have formally constructed a premeasure (see below) on (the left half of) $\mathbb{P}^{1}(\mathbb{R})$, extendable to a 
pseudomeasure on this half with values in the space of holomorphic functions on $\mathbb{C}^{\prime}$, in view of [Manin and Marcolli 2008, Theorem 1.8].

The weak point of this reasoning, about which the word "formally" is supposed to warn the reader, is this: the functions $\chi_{(\alpha, \beta)}(t)(z-t)^{-2 s}$ generally do not belong to the space of test functions as defined in [Lewis and Zagier 1997, p. 225]. Therefore the integrals on the right-hand sides of (1.5) and (1.6) a priori make no sense.

Our heuristic reasoning is in fact a simple extension of the formal argument of [Lewis and Zagier 1997, p. 222], "proving" the three-term functional equation for $\psi(z)$.

In the next subsections, we will provide a precise construction of the pseudomeasures, whose values on the intervals considered above are given by

$$
\mu\left(g^{-1}(-\infty), g^{-1}(0)\right)(z):=(c z+d)^{-2 s} \psi(g z)
$$

without appealing to the integral representation (1.6), but making use of the theory developed in [Lewis and Zagier 1997].

1.2. Preliminaries: left primitive segments. We recall some notions and facts from [Manin and Marcolli 2008]. We consider $\mathbb{Q} \subset \mathbb{R} \subset \mathbb{C}$ as points of an affine line with a fixed coordinate $z$. Completing this line by one point $\infty=-\infty=i \infty$, we get points of the projective line $\mathbb{P}^{1}(\mathbb{Q}) \subset \mathbb{P}^{1}(\mathbb{R}) \subset \mathbb{P}^{1}(\mathbb{C})$ (Riemannian sphere). The group $\operatorname{GL}(2, \mathbb{C})$ acts on $\mathbb{P}^{1}(\mathbb{C})$ by fractional linear transformations. Segments are defined as nonempty connected subsets of $\mathbb{P}^{1}(\mathbb{R})$. A segment is called infinite if $\infty$ is in its closure; otherwise it is called finite. The boundary of each segment generally consists of an unordered pair of points $(\alpha, \beta)$ in $\mathbb{P}^{1}(\mathbb{R})$. We will identify a segment with the ordered pair of its ends: the additional element of structure is its orientation from $\alpha$ to $\beta$. For our purposes, it is usually inessential whether one or two boundary points belong to the segment. In this section we will consider mostly left segments, that is, ones for which $-\infty \leq \alpha$ and $\beta \leq 0$. One-point segments are sometimes called improper ones.

A segment is called rational if its ends are in $\mathbb{P}^{1}(\mathbb{Q})$, and primitive, or Farey, if it is of the form $(g(\infty), g(0))$ for some $g \in \mathrm{GL}(2, \mathbb{Z})$.

A pseudomeasure with values in an abelian group $W$ is a finitely additive $W$ valued function on the boolean algebra of rational segments, vanishing on improper segments. We extended it to oriented segments by the condition that $\mu(\alpha, \beta)=$ $-\mu(\beta, \alpha)$.

In this section, we will construct pseudomeasures supported by left segments. Each such pseudomeasure is defined by its restriction to the set $P$ of positively oriented left primitive segments. We will use the following enumeration of the latter. 
Denote by $S \subset \operatorname{SL}(2, \mathbb{Z})$ the subsemigroup of matrices with nonnegative entries $a, b, c$, and $d$. For any $g \in S,\left(g^{-1}(-\infty), g^{-1}(0)\right)$ is in $P$. In fact, if $c \neq 0$,

$$
g^{-1}(\infty)=\frac{d}{-c}<g^{-1}(0)=\frac{-b}{a}
$$

because $a d-b c=1$. If $c=0$, then $a=d=1$, and again

$$
g^{-1}(\infty)=-\infty<g^{-1}(0)=-b .
$$

Finally, the case $a=0$ does not occur in $S$.

One easily sees that this map $S \rightarrow P: g \mapsto\left(g^{-1}(-\infty), g^{-1}(0)\right)$ is in fact a bijection.

1.3. Preliminaries: the slash operators of complex weight. Here we summarize the considerations of [Lewis and Zagier 2001, p. 240] and [Hilgert et al. 2005, Section 3]. They determine a partial map

$$
\left.(\varphi, g) \mapsto \varphi\right|_{s} g,
$$

allowing us to make sense of and correctly calculate expressions such as those appearing in (1.4) and (1.6). For proofs, see [Hilgert et al. 2005].

(i) Definition domain. The argument $\varphi=\varphi(z)$ in (1.9) can be an arbitrary function holomorphic in some domain of the form $\mathbb{C} \backslash(-\infty, r], r \in \mathbb{R}$. Such functions form a $\mathbb{C}$-algebra which we will denote $\mathscr{F}$. Period functions $\psi=\psi_{s}$ belong to $\mathscr{F}$.

Hilgert et al. call any point $r$ such that $\varphi \in \mathscr{F}$ is holomorphic in $\mathbb{C} \backslash(-\infty, r]$ a branching point of $\varphi$.

The argument $g$ in (1.9) can be any (2,2)-matrix $g=\left(\begin{array}{ll}a & b \\ c & d\end{array}\right)$ with integer entries and nonzero determinant such that either $c>0$ or else $c=0$ and $a, d>0$. Denote by $\mathscr{G}$ the set of such matrices. The set $S$ describing left primitive segments in Section 1.2 is a subset of $\mathscr{G}$. When $g \in \mathscr{G}$ and $s \in \mathbb{C}$, the function $(c z+d)^{s}$ belongs to $\mathscr{F}$.

A pair $(\varphi, g) \in \mathscr{F} \times \mathscr{G}$ belongs to the definition domain $\mathscr{D} \mathscr{Y}$ of the slash operator (1.9) if $\varphi$ admits a branching point $r$ such that either $a-c r>0$, or $a-c r=0$ and $d r-b<0$. For a period function $\varphi=\psi$, we can take $r=0$, and $g$ will do if $a>0$ or if $a=0$ and $b>0$.

Let $\varphi^{+}$be the set of matrices in $\varphi$ such that $b, d \geq 0$ and either $a>0$, or $a=0$ and $b>0$. Again, $S \subset \mathscr{G}^{+}$. Denote by $\mathscr{F}_{0}$ the subspace of $\mathscr{F}_{F}$ admitting 0 as a branch point. Then $\mathscr{F}_{0} \times \mathscr{G}^{+} \subset \mathscr{D}^{\mathcal{S}}$.

(ii) Slash operator of weight $s$. It is the map $\mathscr{D} \mathscr{S} \rightarrow \mathscr{F}$ defined by

$$
(\varphi(z), g) \mapsto\left(\left.\varphi\right|_{s} g\right)(z):=|\operatorname{det} g|^{s}(c z+d)^{-2 s} \varphi(g z) .
$$

It is well defined. Moreover, it sends $\mathscr{F}_{0} \times \mathscr{G}^{+}$to $\mathscr{F}_{0}$. 
(iii) Properties of the slash operator. The basic property is that slash operator is an honest action: if $g_{1}, g_{2} \in \mathscr{G}$ and $\left(\varphi, g_{1}\right),\left(\left.\varphi\right|_{s} g_{1}, g_{2}\right),\left(\varphi, g_{1} g_{2}\right) \in \mathscr{D} \mathscr{Y}$, then

$$
\left.\varphi\right|_{s}\left(g_{1} g_{2}\right)=\left.\left(\left.\varphi\right|_{s} g_{1}\right)\right|_{s} g_{2} \text {. }
$$

(Formally, it is the associativity of the triple product of $\varphi, g_{1}, g_{2}$.) Applying this to $\mathscr{F}_{0} \times \mathscr{G}^{+}$, one can check that $\left.\right|_{s}$ defines a right action of the multiplicative semigroup $\mathscr{G}^{+}$on $\mathscr{F}_{0}$ [Hilgert et al. 2005, Remark 3.4].

From Section 1.2 one sees that if $\left(g^{-1}(-\infty), g^{-1}(0)\right)$ is a left primitive segment, then $g \in \mathscr{G}^{+}$. Since $\psi \in \mathscr{F}_{0}$ in the first equality of (1.6), this expression for $\mu(\alpha, \beta)(z)$ (disregarding the second equality and the poorly defined integral) makes sense, and the slash action can be further iterated.

1.4. The premeasures related to period-like functions. Choose a complex number $s$ and a function $\psi(z) \in \mathscr{F}_{0}$ satisfying the three term functional equation

$$
\psi(z)=\psi(z+1)+(z+1)^{-2 s} \psi\left(\frac{z}{z+1}\right) .
$$

Thus, $\psi$ is a period-like function in the sense of [Lewis and Zagier 2001, Chapter III].

For a left primitive segment $(\alpha, \beta)=\left(g^{-1}(-\infty), g^{-1}(0)\right)$, put

$$
\tilde{\mu}(\alpha, \beta)(z)=(c z+d)^{-2 s} \psi(g z)=\left.\psi\right|_{s}(z) .
$$

Consider now the three left primitive segments $(\alpha, \beta)=\left(g_{1}^{-1}(-\infty), g_{1}^{-1}(0)\right)$, $(\beta, \gamma)=\left(g_{2}^{-1}(-\infty), g_{2}^{-1}(0)\right)$, and $(\alpha, \gamma)=\left(g_{3}^{-1}(-\infty), g_{3}^{-1}(0)\right)$. In plain words, the third segment is broken into two others by a point $\beta$ in the middle.

Lemma 1.4.1. We have

$$
\tilde{\mu}(\alpha, \beta)(z)+\tilde{\mu}(\beta, \gamma)(z)=\tilde{\mu}(\alpha, \gamma)(z) .
$$

Proof. Case 1. $(\alpha, \beta, \gamma)=(-\infty,-1,0)$. In this case

$$
g_{1}=T:=\left(\begin{array}{ll}
1 & 1 \\
0 & 1
\end{array}\right), \quad g_{2}=T^{\prime}:=\left(\begin{array}{ll}
1 & 0 \\
1 & 1
\end{array}\right), \quad g_{3}=I:=\left(\begin{array}{ll}
1 & 0 \\
0 & 1
\end{array}\right),
$$

and (1.13) coincides with (1.11) which can be written as

$$
\left.\psi\right|_{s} I=\left.\psi\right|_{s} T+\left.\psi\right|_{s} T^{\prime} .
$$

Case 2. $g_{1}=T g, g_{2}=T^{\prime} g$, and $g_{3}=g$, where $g \in \operatorname{SL}(2, \mathbb{Z})$ is a matrix with nonnegative entries. In this case, (1.13) reads

$$
\left.\psi\right|_{s} g=\left.\psi\right|_{s} T g+\left.\psi\right|_{s} T^{\prime} g,
$$

which obviously holds in view of (1.14) and the associativity of the slash operator restricted to $\mathscr{F}_{0} \times \mathscr{G}^{+}$. 
General case. In fact, the previous case is general: we necessarily have $g_{1}=T g_{3}$ and $g_{2}=T^{\prime} g_{3}$.

Let us check this for the case when $\alpha \neq-\infty$ leaving the remaining case to the reader. Put $\alpha=d /-c$ and $\gamma=-b / a$ as in Section 1.2 where $(a, b, c, d)$ are the entries of $g_{3}$. Then the only possible value of $\beta$ is $\beta=-(b+d) /(a+c)=$ $(b+d) /-(a+c)$ as is well known from the classical theory of Farey series. This fact directly translates into $g_{1}=T g_{3}$ and $g_{2}=T^{\prime} g_{3}$.

Remark. Notice that if $\psi(z)$ is an actual period function for a Maass wave form, the lemma becomes obvious in view of the integral representation of $\psi(z)$ proven in [Lewis and Zagier 2001, Chapter II, Section 1]. The relevant formula on p. 212 of that reference, with $\psi_{1}$ replace by $\psi$ and a change in sign, is

$$
(c \zeta+d)^{-2 s} \psi(g \zeta)=\int_{g^{-1}(\infty)}^{g^{-1}(0)}\left\{u, R_{\zeta}^{s}\right\}(z) .
$$

In this formula, we integrate a closed form along an arbitrary path leaving $\zeta$ and $\bar{\zeta}$ to the right of it. Additivity (1.13) becomes evident.

We will use this integral representation in the next section.

1.4.2. The premeasure on left segments. To define a premeasure in the sense of [Manin and Marcolli 2008], supported by the subset of left primitive segments, it remains to complete (1.12) of the function $\tilde{\mu}$ by putting for $\alpha<\beta \leq 0$

$$
\tilde{\mu}(\beta, \alpha):=-\tilde{\mu}(\alpha, \beta), \quad \tilde{\mu}(\alpha, \alpha)=0 .
$$

One easily checks that (1.13) continues to hold on this extended domain.

1.5. The pseudomeasure related to a period-like function. Now we can state the main result of this section.

Theorem 1.5.1. There exists a unique finitely additive function $\mu$ with values in $\mathscr{F}_{0}$ coinciding with $\tilde{\mu}$ on left primitive segments and vanishing on all rational segments in $(0, \infty)$.

Sketch of proof. We recall the plan of the proof of [Manin and Marcolli 2008, Theorem 1.8]. It consists of the following steps.

(1) Using the "continued fractions trick", we show that for any nonpositive rational (or infinite) $\alpha$ and $\beta$ one can find a sequence of rational nonpositive numbers $\alpha_{0}=\alpha, \alpha_{1}, \ldots, \alpha_{n}=\beta$ such that $\left(\alpha_{i}, \alpha_{i+1}\right)$ is a left primitive segment for all $i=0, \ldots, n-1$. Such a sequence is called a primitive chain connecting $\alpha$ to $\beta$.

(2) Having chosen such a primitive chain, we put 


$$
\mu(\alpha, \beta):=\sum_{i=0}^{n-1} \tilde{\mu}\left(\alpha_{i}, \alpha_{i+1}\right) .
$$

(3) The fact that (1.16) does not depend on the choice of the connecting primitive chain is checked by proving that any two chains can be transformed one to another by using "elementary moves" compatible with relations that hold for $\tilde{\mu}$. An elementary move essentially replaces a Farey interval $\left(\frac{a}{c}, \frac{b}{d}\right)$ by the chain $\left(\frac{a}{c}, \frac{a+b}{c+d}\right),\left(\frac{a+b}{c+d}, \frac{b}{d}\right)$, or vice versa.

(4) Finally, we have to check that (1.16) implies finite additivity and the sign change after the change of orientation. This is straightforward.

1.6. Modularity. Let $\Gamma$ be a subgroup of $\operatorname{SL}(2, \mathbb{Z})$ and $W$ a left $\Gamma$-module.

In [Manin and Marcolli 2008], a pseudomeasure $\mu$ with values in $W$ is called $\Gamma$-modular if for all $g \in \Gamma$ and $\alpha, \beta \in \mathbb{P}^{1}(\mathbb{Q})$ we have

$$
\mu(g \alpha, g \beta)=g \mu(\alpha, \beta) .
$$

It was checked that such pseudomeasures correspond to parabolic 1-cocycles.

In our context, this is replaced by the following property: for all $g$ with $g^{-1} \in S$ and any left segment $(\alpha, \beta)$,

$$
\mu\left(g^{-1}(\alpha), g^{-1}(\beta)\right)=\left.\mu(\alpha, \beta)\right|_{s} g
$$

In fact, it suffices to check this for left primitive segments, say

$$
(\alpha, \beta)=\left(h^{-1}(-\infty), h^{-1}(0)\right),
$$

in which case we have

$$
\begin{aligned}
\mu\left(g^{-1}(\alpha), g^{-1}(\beta)\right) & =\mu\left((h g)^{-1}(-\infty),(h g)^{-1}(0)\right) \\
& =\left.\psi\right|_{s}(h g)=\left.\left(\psi \mid{ }_{s} h\right)\right|_{s} g=\left.\mu(\alpha, \beta)\right|_{s} g .
\end{aligned}
$$

Since the right slash action of $g$ can be considered as the left action of $g^{-1}$, we can say that (1.17) expresses the modularity of $\mu$ with respect to the multiplicative semigroup $S^{-1} \subset \operatorname{SL}(2, \mathbb{Z})$.

\section{Maass $L$-functions and their Mellin-Lévy transforms}

2.1. Maass L-series as sums over rational numbers. Let $u=u_{s}$ be a Maass cusp form, which is an eigenfunction with respect to all Hecke operators

$$
T_{m}:=\sum_{\substack{a d=m \\
0<b \leq d}}\left(\begin{array}{rr}
a & -b \\
0 & d
\end{array}\right)
$$

acting via the slash operator of weight $0:\left.u \mapsto u\right|_{0} T_{m}=\lambda_{m} u$. 
Put

$$
L_{u}(\rho):=\sum_{m=1}^{\infty} \frac{\lambda_{m}}{m^{\rho}} .
$$

The action of the Hecke operators on $u$ induces an action on the period functions, which can be explicitly described by a nice formula, for example, as in [Mühlenbruch 2004]. However, we will need a different expression, involving the pseudomeasure $\mu_{u}$, and we will start with an heuristic derivation of it, as in Section 1.1 .

Let us formally apply the slash operator $\left.\right|_{-s}$ of (1.10) to the boundary measure $U(t) d t$ and denote the resulting action upon the respective period function $\psi$ by $T_{m}^{*}$. In this heuristic calculation we "define" $\psi$ by (1.1). The choice of weight $-s$ is motivated by the invariance property (1.2). We get

$$
\begin{aligned}
\left(\psi \mid T_{m}^{*}\right)(\zeta) & :=\int_{-\infty}^{0}(\zeta-t)^{-2 s}\left(\left.U(t) d t\right|_{-s} T_{m}\right) \\
& =\sum_{\substack{a d=m \\
0<b \leq d}}\left(\frac{d}{a}\right)^{s} \int_{-\infty}^{0}(\zeta-t)^{-2 s} U\left(\frac{a t-b}{d}\right) d\left(\frac{a t-b}{d}\right) .
\end{aligned}
$$

Make the change of variable $\tau=\frac{a t-b}{d}$. The last integral takes the form

$$
\begin{aligned}
\sum_{\substack{a d=m \\
0<b \leq d}}\left(\frac{d}{a}\right)^{s} \int_{-\infty}^{-b / d}\left(\zeta-\frac{d \tau+b}{a}\right)^{-2 s} U(\tau) d \tau & \\
= & \sum_{\substack{a d=m \\
0<b \leq d}}\left(\frac{d}{a}\right)^{s} \int_{-\infty}^{-b / d}\left(\frac{d z+b}{a}-\frac{d \tau+b}{a}\right)^{-2 s} U(\tau) d \tau,
\end{aligned}
$$

where $z=\frac{a \zeta-b}{d}$. The integral in the last sum can be rewritten as

$$
\left(\frac{a}{d}\right)^{2 s} \int_{-\infty}^{-b / d}(\zeta-\tau)^{-2 s} U(\tau) d \tau
$$

Thus, heuristically,

$$
\begin{aligned}
\left(\psi \mid T_{m}^{*}\right)(\zeta) & =\left(\mu(-\infty, 0) \mid T_{m}^{*}\right)(\zeta)=\sum_{\substack{a d=m \\
0<b \leq d}}\left(\frac{a}{d}\right)^{s} \mu\left(-\infty,-\frac{b}{d}\right)\left(\frac{a \zeta-b}{d}\right) \\
& =\left.\sum_{\substack{a d=m \\
0<b \leq d}} \mu\left(-\infty,-\frac{b}{d}\right)\right|_{s}\left(\begin{array}{rr}
a & -b \\
0 & d
\end{array}\right)(\zeta) .
\end{aligned}
$$

This expression is useful for our purposes because it allows us to represent the (somewhat normalized) Dirichlet series $L_{u}(s)$ as a natural sum over rational numbers. We will state now the respective theorem: 
Theorem 2.2. We have

$$
\begin{aligned}
\psi(z) \sum_{m=1}^{\infty} \frac{\lambda_{m}}{m^{\rho}} & \\
& =\left.\zeta(\rho-s) \zeta(\rho+s) \sum_{q=1}^{\infty} \frac{1}{q^{\rho}} \sum_{\substack{0<p \leq q \\
(p, q)=1}} \mu(-\infty,-p / q)\right|_{s}\left(\begin{array}{rr}
1 & -p \\
0 & q
\end{array}\right)(z) .
\end{aligned}
$$

Proof. Step 1. First, we have to supply an honest proof of (2.3). In [Lewis and Zagier 2001, Chapter II, Section 2], the authors construct a differential 1-form $\left\{u, R_{\zeta}^{s}\right\}(z)$ which we invoked at the end of Lemma 1.4.1. It has the following properties:

(i) $\left\{u, R_{\zeta}^{s}\right\}(z)$ is a closed smooth form of $z$ varying in the complex upper half-plane $H$. It depends on the parameter $\zeta \in \mathbb{C}$ holomorphically when $z \neq \zeta, \bar{\zeta}$. Generally it is multivalued, but a well-defined branch can be chosen on the complement in $H$ of a path joining $\zeta$ to $\bar{\zeta}$.

(ii) The period function $\psi(\zeta), \zeta \in H$ for $u$ (up to a constant proportionality factor) can be then written as an integral:

$$
\psi(\zeta)=\int_{-\infty}^{0}\left\{u, R_{\zeta}^{s}\right\}(z)
$$

taken along any path in $H$ leaving $\zeta$ to the left of it.

Now assume that $\left.u\right|_{0} T_{m}=\lambda_{m} u$ for $T_{m}$ from (2.1) and a constant $\lambda_{m}$. Then we have from (2.5) and (2.1)

$$
\lambda_{m} \psi(\zeta)=\int_{-\infty}^{0}\left\{\sum_{\substack{a d=m \\ 0<b \leq d}} u\left(\frac{a z-b}{d}\right), R_{\zeta}^{s}\right\}(z) .
$$

For each $a, b$, and $d$ fixed, we first want to make the implicit argument $z$ of $R_{\zeta}^{s}$ the same as that of $u$, i.e., $(a z-b) / d$. We have (see [Lewis and Zagier 2001, p. 211]):

$$
\begin{aligned}
R_{\zeta}(z)= & \frac{i}{2}\left((z-\zeta)^{-1}-(\bar{z}-\zeta)^{-1}\right)= \\
& \frac{a}{d} \cdot \frac{i}{2}\left(\left(\frac{a z-b}{d}-\frac{a \zeta-b}{d}\right)^{-1}-\left(\frac{a \bar{z}-b}{d}-\frac{a \zeta-b}{d}\right)^{-1}\right)=\frac{a}{d} R_{\xi}\left(\frac{a z-b}{d}\right),
\end{aligned}
$$

where $\xi:=(a \zeta-b) / d$.

Substituting this into (2.6), we obtain

$$
\lambda_{m} \psi(\zeta)=\sum_{\substack{a d=m \\ 0<b \leq d}}\left(\frac{a}{d}\right)^{s} \int_{-\infty}^{0}\left\{u\left(\frac{a z-b}{d}\right), R_{(a \zeta-b) / d}^{s}\left(\frac{a z-b}{d}\right)\right\} .
$$


Considering now $z \mapsto(a z-b) / d$ as a holomorphic change of variables, we infer from [Lewis and Zagier 2001, Lemma, p. 210] that the integrand in the respective term of (2.7) can be rewritten as

$$
\left\{u, R_{(a \zeta-b) / d}^{s}\right\}\left(\frac{a z-b}{d}\right) .
$$

Hence, finally,

$$
\begin{aligned}
\lambda_{m} \psi(\zeta) & =\sum_{\substack{a d=m \\
0<b \leq d}}\left(\frac{a}{d}\right)^{s} \int_{-\infty}^{-b / d}\left\{u, R_{(a \zeta-b) / d}^{s}\right\}(z) \\
& =\left.\sum_{\substack{a d=m \\
0<b \leq d}} \mu(-\infty,-b / d)\right|_{s}\left(\begin{array}{rr}
a & -b \\
0 & d
\end{array}\right)(\zeta) .
\end{aligned}
$$

This is formula (2.3), written for $u$ which is an eigenfunction of $T_{m}$, and its respective period function.

Step 2. Multiply (2.8) by $m^{-\rho}$ and sum over all $m=1,2, \ldots$ Again replacing the free variable $\zeta$ by $z$, to avoid confusion with Riemann's zeta, we obtain

$$
\psi(z) \sum_{m=1}^{\infty} \frac{\lambda_{m}}{m^{\rho}}=\left.\sum_{m=1}^{\infty} \frac{1}{m^{\rho}} \sum_{\substack{a d=m \\
0<b \leq d}} \mu(-\infty,-b / d)\right|_{s}\left(\begin{array}{rr}
a & -b \\
0 & d
\end{array}\right)(z) .
$$

Each matrix in (2.9) can be uniquely written in the following way:

$$
\left(\begin{array}{rr}
a & -b \\
0 & d
\end{array}\right)=\left(\begin{array}{rr}
d_{2} & -p d_{1} \\
0 & q d_{1}
\end{array}\right)=\left(\begin{array}{rr}
1 & -p \\
0 & q
\end{array}\right)\left(\begin{array}{ll}
1 & 0 \\
0 & d_{1}
\end{array}\right)\left(\begin{array}{ll}
d_{2} & 0 \\
0 & 1
\end{array}\right)
$$

where $m=d_{1} d_{2} q, d_{i} \geq 1$, and $0<p \leq q,(p, q)=1$. Moreover, the arbitrary quadruple $\left(d_{1}, d_{2}, p, q\right)$ satisfying these conditions produces one term in (2.9).

From (2.10) and the associativity of the slash operator (1.10) it follows that

$$
\left.\right|_{s}\left(\begin{array}{rr}
a & -b \\
0 & d
\end{array}\right)=\left.\right|_{s}\left(\begin{array}{rr}
1 & -p \\
0 & q
\end{array}\right) \cdot d_{1}^{-s} d_{2}^{s} \text {. }
$$

Hence we can rewrite (2.9) as follows:

$$
\psi(z) \sum_{m=1}^{\infty} \frac{\lambda_{m}}{m^{\rho}}=\left.\sum_{q, d_{1}, d_{2}=1}^{\infty} \frac{1}{q^{\rho} d_{1}^{\rho-s} d_{2}^{\rho+s}} \sum_{\substack{0<p \leq q \\
(p, q)=1}} \mu(-\infty,-p / q)\right|_{s}\left(\begin{array}{rr}
1 & -p \\
0 & q
\end{array}\right)(z) .
$$

This last expression is seen to be equal to the right-hand side of (2.4), concluding the proof. 
2.3. The Lévy-Mellin transform. Now put

$$
r_{u}(p, q):=\left.(p+q) q^{1-\rho} \mu(-\infty,-p / q)\right|_{s}\left(\begin{array}{rr}
1 & -p \\
0 & q
\end{array}\right)(z) \cdot \psi(z)^{-1}
$$

and

$$
l_{u}(\xi):=\sum_{i=0}^{\infty} \sum_{i=0}^{\infty} r\left(q_{i}(\xi), q_{i+1}(\xi)\right) .
$$

From (2.4) and (0.18) we get:

Corollary 2.3.1. Let $u$ be a Maass cusp form, $\Delta u=s(1-s) u$, and $u \mid T_{m}=\lambda_{m} u$ for all $m \geq 1$. Put

$$
\Lambda_{u}(\rho):=\zeta(\rho-s)^{-1} \zeta(\rho+s)^{-1} \sum_{m=1}^{\infty} \frac{\lambda_{m}}{m^{\rho}} .
$$

Then

$$
\Lambda_{u}(\rho)=\int_{0}^{1 / 2} l_{u}(\xi) d \xi
$$

Remark 2.3.2. The class of series of the form (0.18) involving modular symbols includes also the Eisenstein series of [Goldfeld 1999]. They certainly deserve further study.

2.4. Hecke operators on period functions via continued fractions. Consider the sequence of normalized convergents $b / d$ as in [Manin and Marcolli 2008, (1.5)]. When $0<b / d<1$, it starts with

$$
-\infty=\frac{1}{0}=: \frac{b_{-1}}{d_{-1}}, \quad 0=\frac{0}{1}=: \frac{b_{0}}{d_{0}}, \ldots, b / d=\frac{b_{n}}{d_{n}},
$$

where $n=n(b / d)$ is the length of the continued fraction expansion.

The following sequence of left primitive segments $I_{k}=I_{k}(b / d)$ connects $-\infty$ to $-b / d$. We order their ends from the left one to the right one, and put a minus before those that should be run in the opposite direction in our chain:

$$
\begin{array}{ll}
I_{0}=(-\infty, 0)=\left(-\frac{b_{-1}}{d_{-1}},-\frac{b_{0}}{d_{0}}\right), & I_{1}=-\left(-\frac{b_{1}}{d_{1}},-\frac{b_{0}}{d_{0}}\right), \\
I_{2}=\left(-\frac{b_{1}}{d_{1}},-\frac{b_{2}}{d_{2}}\right), & I_{3}=-\left(-\frac{b_{3}}{d_{3}},-\frac{b_{2}}{d_{2}}\right),
\end{array}
$$

and generally

$$
I_{k}=(-1)^{k}\left(-\frac{b_{k-\varepsilon_{k}}}{d_{k-\varepsilon_{k}}},-\frac{b_{k-\varepsilon_{k+1}}}{d_{k-\varepsilon_{k+1}}}\right)
$$

where $\varepsilon_{k}=1$ for even $k$ and $\varepsilon_{k}=0$ for odd $k$.

This means that

$$
(-1)^{k} I_{k}=\left(g_{k}^{-1}(-\infty), g_{k}^{-1}(0)\right)
$$


where

$$
g_{k}=g_{k, b / d}=\left(\begin{array}{cc}
d_{k-\varepsilon_{k+1}} & b_{k-\varepsilon_{k+1}} \\
d_{k-\varepsilon_{k}} & b_{k-\varepsilon_{k}}
\end{array}\right) \in S .
$$

Therefore, (2.8) can be rewritten as

$$
\lambda_{m} \psi(\zeta)=\sum_{\substack{a d=m \\ 0<b \leq d}}\left(\frac{a}{d}\right)^{s} \sum_{k=0}^{n(b / d)}(-1)^{k} \int_{-\infty}^{0}\left\{u\left(g_{k, b / d}(z)\right), R_{(a \zeta-b) / d}^{s}\left(g_{k, b / d}(z)\right)\right\} .
$$

We have $u\left(g_{k, b / d}(z)\right)=u(z)$ and

$$
R_{(a \zeta-b) / d}^{s}\left(g_{k, b / d}(z)\right)=\left(d_{k-\varepsilon_{k}} g_{k, b / d}^{-1}\left(\frac{a \zeta-b}{d}\right)+b_{k-\varepsilon_{k}}\right)^{2 s} R_{g_{k, b / d}^{-1}((a \zeta-b) / d)}^{s}(z) .
$$

This follows from the formula (2.6) on [Lewis and Zagier 2001, p. 211] and (2.13). To shorten notation, set

$$
j_{k}(b / d, \zeta)^{2 s}:=\left(d_{k-\varepsilon_{k}} g_{k, b / d}^{-1}\left(\frac{a \zeta-b}{d}\right)+b_{k-\varepsilon_{k}}\right)^{2 s} .
$$

Then we get

$$
\begin{aligned}
\lambda_{m} \psi(\zeta) & =\sum_{\substack{a d=m \\
0<b \leq d}}\left(\frac{a}{d}\right)^{s} \sum_{k=0}^{n(b / d)}(-1)^{k} j_{k}(b / d, \zeta)^{2 s} \int_{-\infty}^{0}\left\{u(z), R_{g_{k, b / d}^{-1}((a \zeta-b) / d)}^{s}(z)\right\} \\
& =\sum_{\substack{a d=m \\
0<b \leq d}}\left(\frac{a}{d}\right)^{s} \sum_{k=0}^{n(b / d)}(-1)^{k} j_{k}(b / d, \zeta)^{2 s} \psi\left(g_{k, b / d}^{-1}\left(\frac{a \zeta-b}{d}\right)\right)
\end{aligned}
$$

In order to deduce from (2.17) a nice explicit formula for $\lambda_{m}$, as was done in [Manin 1973] for the coefficients of the classical cusp forms, one could use an appropriate linear functional on functions of $\zeta$. In the classical case, it was the highest coefficient (or the constant term) of the period polynomial.

In the Maass case, one could try to use asymptotic behaviors at 0 or $\infty$. Other forms of Hecke operators, as (2.18), might be useful.

2.5. Hecke operators and transfer operator. In [Mühlenbruch 2004] it is shown, using the method of [Choie and Zagier 1993], that the Hecke operators acting on period functions for the full modular group can be written in the nice form

$$
T_{m}^{+}=\sum_{\substack{a>c \geq 0 \\
d>b \geq 0 \\
a d-b c=m}}\left(\begin{array}{ll}
a & b \\
c & d
\end{array}\right)
$$

Of course, they act on $\psi(z)$ via $\left.\right|_{s}$ in our notation. (Mühlenbruch denotes this slash operator by $\left.\right|_{2 s}$.) In particular, for $m=1$ we have $T_{m}^{+}=I$. 
However, if we change the summation domain slightly, replacing $a>c \geq 0$ by $a \geq c>0$, then the equations for case $m=1$ will admit the following solutions. From $a d=1+b c \leq 1+(d-1) a$ it follows that $a=c=1$ and $d=b+1 \geq 1$ so that we will get the operator

$$
T_{1}^{*}:=\sum_{b=0}^{\infty}\left(\begin{array}{cc}
1 & b \\
1 & b+1
\end{array}\right) .
$$

This correction is not as ad hoc as it seems. In fact, if we compare it with the Atkin-Lehner operators for the group $\Gamma_{0}(N)$ and $p / N$,

$$
U_{p}:=T_{p}-p \cdot \mathrm{id},
$$

we will see that $T_{1}^{*}$ imitates the "characteristic 1" Atkin-Lehner operator corresponding to the "improper prime $p=1$ ", with eigenvalue 1 on $\psi$ :

Claim 2.5.1. If $\psi(z)$ is a period function for a Maass cusp form of weight $s$ with $\operatorname{Re} s>0, s \neq \frac{1}{2}$, then

$$
\left.\psi\right|_{s} T_{1}^{*}(z)=\psi(z)
$$

Proof. Assume moreover that

$$
\psi^{\tau}(z):=\left.\psi\right|_{s}\left(\begin{array}{cc}
0 & 1 \\
1 & 0
\end{array}\right)(z)=\varepsilon \psi(z), \quad \varepsilon= \pm 1,
$$

so that $\psi$ is even or odd. This is not a restriction because any $\psi$ is the sum of an even and an odd period function.

According to [Lewis and Zagier 2001, p. 255], the function

$$
h(z):=\psi(z+1)=\left.\psi\right|_{s}\left(\begin{array}{ll}
1 & 1 \\
0 & 1
\end{array}\right)(z)
$$

satisfies the equation

$$
\left.\varepsilon h\right|_{s}\left(\sum_{n=1}^{\infty}\left(\begin{array}{ll}
0 & 1 \\
1 & n
\end{array}\right)\right)(z)=h(z) .
$$

Substituting first (2.22) into (2.23), and then (2.21) into the resulting identity, yields

$$
\left.\left.\left.\psi\right|_{s}\left(\begin{array}{ll}
0 & 1 \\
1 & 0
\end{array}\right)\right|_{s}\left(\begin{array}{ll}
1 & 1 \\
0 & 1
\end{array}\right)\right|_{s}\left(\sum_{n=1}^{\infty}\left(\begin{array}{ll}
0 & 1 \\
1 & n
\end{array}\right)\right)(z)=\left.\psi\right|_{s}\left(\begin{array}{ll}
1 & 1 \\
0 & 1
\end{array}\right)(z) .
$$

The associativity of the slash operator and the identity

$$
\left(\begin{array}{ll}
0 & 1 \\
1 & 0
\end{array}\right)\left(\begin{array}{ll}
1 & 1 \\
0 & 1
\end{array}\right)\left(\begin{array}{ll}
0 & 1 \\
1 & n
\end{array}\right)\left(\begin{array}{rr}
1 & -1 \\
0 & 1
\end{array}\right)=\left(\begin{array}{cc}
1 & n-1 \\
0 & n
\end{array}\right)
$$

establish (2.20). 
2.6. The Brjuno function and derivatives of the classical L-functions. The Brjuno function of (0.20) is defined in this context as a generalized Lévy sum

$$
B(\xi):=\sum_{j=0}^{\infty}\left|p_{j}(\xi)-q_{j}(\xi) \xi\right| \log \frac{p_{j-1}(\xi)-q_{j-1}(\xi) \xi}{q_{j}(\xi) \xi-p_{j}(\xi)} .
$$

This series diverges on a set of measure 0 . Outside it converges to a measurable function, continuous at irrational points, with period 1 [Marmi et al. 2006].

The values of derivatives of Mellin transforms of classical forms were studied by D. Goldfeld [1995] and Diamantis [1999]. Goldfeld's idea consisted in replacing the $\log y$ initially appearing in the Mellin expression for the first derivative by the logarithm of the $\eta$-function, or a combination of such, to enhance the modular properties of the integrand. The same game can be played with the Brjuno function in place of the $\eta$-function.

Consider a classical cusp form $u(z)$ for $\operatorname{SL}(2, Z)$ of integral weight $2 k=w+2$ as on page 1092. Let $L_{u}(s)$ be its Mellin transform.

Proposition 2.6.1. We have

$$
L_{u}^{\prime}(w / 2+2)=C\left(-\int_{0}^{1} u(i y) y^{w / 2} B(y) d y+\int_{1}^{\infty} u(i y) y^{w / 2-1} B(y) d y\right),
$$

where

$$
C=\frac{(2 \pi)^{(w+4) / 2}}{\Gamma((w+2) / 2)}\left(1+i^{w+2}\right) .
$$

Proof. An easy calculation shows that $B(\xi)$ satisfies the functional equation

$$
B(\xi)=-\log \xi+\xi B\left(\xi^{-1}\right), \quad \xi \in(0,1) .
$$

Therefore, we have

$$
\begin{aligned}
& \int_{0}^{\infty} u(i y) y^{w / 2} \log y d y \\
& \quad=\int_{0}^{1} u(i y) y^{w / 2}\left(-B(y)+y B\left(y^{-1}\right)\right) d y+\int_{1}^{\infty} u(i v) v^{w / 2}\left(v^{-1} B(v)-B\left(v^{-1}\right)\right) d v .
\end{aligned}
$$

In the second summand of the second integrand, make the change of variable $v=$ $y^{-1}$, and combine it with the first summand of the first integrand. Similarly, in the second summand of the first integrand, make the change of variable $y=v^{-1}$, and combine it with the first summand of the second integrand. This will result in

$$
\left(1+i^{w+2}\right)\left(-\int_{0}^{1} u(i y) y^{w / 2} B(y) d y+\int_{1}^{\infty} u(i y) y^{w / 2-1} B(y) d y\right) .
$$

The remaining factor in $C$ comes from the Mellin transform. 
Acknowledgement. I am grateful to Don Zagier, who read a preliminary draft of this paper and suggested a number of corrections and complements.

\section{References}

[Choie and Zagier 1993] Y. Choie and D. Zagier, "Rational period functions for PSL(2, Z)", pp. 89-108 in A tribute to Emil Grosswald: number theory and related analysis, edited by M. Knopp and M. Sheingorn, Contemp. Math. 143, Amer. Math. Soc., Providence, RI, 1993. MR 94g:11033 Zbl 0790.11044

[Diamantis 1999] N. Diamantis, "Special values of higher derivatives of $L$-functions", Forum Math. 11:2 (1999), 229-252. MR 2000a:11075 Zbl 0932.11032

[Goldfeld 1995] D. Goldfeld, "Special values of derivatives of $L$-functions", pp. 159-173 in Number theory (Halifax, NS, 1994), edited by K. Dilcher, CMS Conf. Proc. 15, Amer. Math. Soc., Providence, RI, 1995. MR 96h:11039 Zbl 0845.11021

[Goldfeld 1999] D. Goldfeld, "Zeta functions formed with modular symbols", pp. 111-121 in Automorphic forms, automorphic representations, and arithmetic (Fort Worth, TX, 1996), edited by R. S. Doran et al., Proc. Sympos. Pure Math. 66, Amer. Math. Soc., Providence, RI, 1999. MR 2000g:11039 Zbl 0934.11026

[Hilgert et al. 2005] J. Hilgert, D. Mayer, and H. Movasati, "Transfer operators for $\Gamma_{0}(n)$ and the Hecke operators for the period functions of PSL(2, ZZ)", Math. Proc. Cambridge Philos. Soc. 139:1 (2005), 81-116. MR 2006c:11057 Zbl 1129.11022

[Lewis and Zagier 1997] J. Lewis and D. Zagier, "Period functions and the Selberg zeta function for the modular group", pp. 83-97 in The mathematical beauty of physics (Saclay, 1996), edited by J. M. Drouffe and J. B. Zuber, Adv. Ser. Math. Phys. 24, World Sci. Publ., River Edge, NJ, 1997. MR 99c:11108 Zbl 1058.11544

[Lewis and Zagier 2001] J. Lewis and D. Zagier, "Period functions for Maass wave forms, I", Ann. of Math. (2) 153:1 (2001), 191-258. MR 2003d:11068 Zbl 1061.11021

[Maass 1949] H. Maass, "Über eine neue Art von nichtanalytischen automorphen Funktionen und die Bestimmung Dirichletscher Reihen durch Funktionalgleichungen", Math. Ann. 121 (1949), 141-183. MR 11,163c Zbl 0033.11702

[Manin 1972] Y. I. Manin, "Parabolic points and zeta functions of modular curves", Izv. Akad. Nauk SSSR Ser. Mat. 36 (1972), 19-66. In Russian; translated in Math. USSR-Izv. 6 (1972), 19-64. MR 47 \#3396 Zbl 0243.14008

[Manin 1973] Y. I. Manin, "The periods of modular forms and p-adic Hecke series", Mat. Sb. (N.S.) 92(134) (1973), 378-401. In Russian; translated in Math. USSR-Sb. 21:3 (1973), 371-393. MR 49 \#10638 Zbl 0293.14007

[Manin 1974] Y. I. Manin, "The values of $p$-adic Hecke series at integer points of the critical strip", Math. USSR, Sb. 22:4 (1974), 631-637. Zbl 0304.14013

[Manin and Marcolli 2008] Y. I. Manin and M. Marcolli, "Modular shadows and the Lévy-Mellin $\infty$-adic transform", pp. 189-238 in Modular forms on Schiermonnikoog, edited by B. Edixhoven et al., Cambridge Univ. Press, 2008. MR 2512362 Zbl 05503855

[Marmi et al. 2001] S. Marmi, P. Moussa, and J.-C. Yoccoz, "Complex Brjuno functions", J. Amer. Math. Soc. 14:4 (2001), 783-841. MR 2003a:37061 Zbl 1051.37021

[Marmi et al. 2006] S. Marmi, P. Moussa, and J.-C. Yoccoz, "Some properties of real and complex Brjuno functions", pp. 601-623 in Frontiers in number theory, physics, and geometry, I, edited by P. Cartier et al., Springer, Berlin, 2006. MR 2009d:37071 Zbl 1134.37018 
[Mayer 1991a] D. H. Mayer, "Continued fractions and related transformations", pp. 175-222 in Ergodic theory, symbolic dynamics, and hyperbolic spaces (Trieste, 1989), edited by T. Bedford et al., Oxford Sci. Publ., Oxford Univ. Press, New York, 1991. MR 1130177 Zbl 0755.58034

[Mayer 1991b] D. H. Mayer, "The thermodynamic formalism approach to Selberg's zeta function for PSL(2, Z)”, Bull. Amer. Math. Soc. (N.S.) 25:1 (1991), 55-60. MR 91j:58130 Zbl 0729.58041

[Mazur and Swinnerton-Dyer 1974] B. Mazur and P. Swinnerton-Dyer, "Arithmetic of Weil curves", Invent. Math. 25 (1974), 1-61. MR 50 \#7152 Zbl 0281.14016

[Mühlenbruch 2004] T. Mühlenbruch, "Hecke operators on period functions for the full modular group”, Int. Math. Res. Not. 2004:77 (2004), 4127-4145. MR 2005k:11089 Zbl 1065.11031

[Shokurov 1980a] V. V. Shokurov, "Shimura integrals of cusp forms", Izv. Akad. Nauk SSSR Ser. Mat. 44:3 (1980), 670-718, 720. MR 82b:10029 Zbl 0444.14030

[Shokurov 1980b] V. V. Shokurov, “A study of the homology of Kuga varieties”, Izv. Akad. Nauk SSSR Ser. Mat. 44:2 (1980), 443-464, 480. In Russian; translated in Math. USSR-Izv. 16:2 (1981), 399-418. MR 82f:14023

[Višik and Manin 1974] M. M. Višik and Y. I. Manin, " $p$-adic Hecke series of imaginary quadratic fields", Mat. Sb. (N.S.) 95(137) (1974), 357-383, 471. In Russian; translated in Math. USSR, Sb. 24 (1976), 345-371. MR 51 \#8078

Communicated by Peter Sarnak

Received 2010-01-12 Accepted 2010-10-01

manin@mpim-bonn.mpg.de Max-Planck-Institut für Mathematik, Vivatsgasse 7, D-53111 Bonn, Germany

Department of Mathematics, Northwestern University,

Evanston, IL 60208-2730, United States 


\section{Algebra \& Number Theory}

www.jant.org

\section{EDITORS}

\section{MANAGING EDITOR}

Bjorn Poonen

Massachusetts Institute of Technology

Cambridge, USA

\author{
EDITORIAL BOARD CHAIR \\ David Eisenbud \\ University of California \\ Berkeley, USA
}

\section{BOARD OF EDITORS}

\section{Georgia Benkart}

Dave Benson

Richard E. Borcherds

John H. Coates

J-L. Colliot-Thélène

Brian D. Conrad

Hélène Esnault

Hubert Flenner

Edward Frenkel

Andrew Granville

Joseph Gubeladze

Ehud Hrushovski

Craig Huneke

Mikhail Kapranov

Yujiro Kawamata

János Kollár

Hendrik W. Lenstra

Yuri Manin

Barry Mazur
University of Wisconsin, Madison, USA

University of Aberdeen, Scotland

University of California, Berkeley, USA

University of Cambridge, UK

CNRS, Université Paris-Sud, France

University of Michigan, USA

Universität Duisburg-Essen, Germany

Ruhr-Universität, Germany

University of California, Berkeley, USA

Université de Montréal, Canada

San Francisco State University, USA

Hebrew University, Israel

University of Kansas, USA

Yale University, USA

University of Tokyo, Japan

Princeton University, USA

Universiteit Leiden, The Netherlands

Northwestern University, USA

Harvard University, USA
Susan Montgomery

Shigefumi Mori

Andrei Okounkov

Raman Parimala

Victor Reiner

Karl Rubin

Peter Sarnak

Michael Singer

Ronald Solomon

Vasudevan Srinivas

J. Toby Stafford

Bernd Sturmfels

Richard Taylor

Ravi Vakil

Michel van den Bergh

Marie-France Vignéras

Kei-Ichi Watanabe

Andrei Zelevinsky

Efim Zelmanov
University of Southern California, USA

RIMS, Kyoto University, Japan

Princeton University, USA

Emory University, USA

University of Minnesota, USA

University of California, Irvine, USA

Princeton University, USA

North Carolina State University, USA

Ohio State University, USA

Tata Inst. of Fund. Research, India

University of Michigan, USA

University of California, Berkeley, USA

Harvard University, USA

Stanford University, USA

Hasselt University, Belgium

Université Paris VII, France

Nihon University, Japan

Northeastern University, USA

University of California, San Diego, USA

\section{PRODUCTION}

ant@mathscipub.org

Silvio Levy, Scientific Editor

Andrew Levy, Production Editor

See inside back cover or www.jant.org for submission instructions.

The subscription price for 2010 is US \$140/year for the electronic version, and \$200/year (+\$30 shipping outside the US) for print and electronic. Subscriptions, requests for back issues from the last three years and changes of subscribers address should be sent to Mathematical Sciences Publishers, Department of Mathematics, University of California, Berkeley, CA 94720-3840, USA.

Algebra \& Number Theory (ISSN 1937-0652) at Mathematical Sciences Publishers, Department of Mathematics, University of California, Berkeley, CA 94720-3840 is published continuously online. Periodical rate postage paid at Berkeley, CA 94704, and additional mailing offices.

ANT peer review and production are managed by EditFLOW ${ }^{\mathrm{TM}}$ from Mathematical Sciences Publishers.

\section{PUBLISHED BY}

mathematical sciences publishers

http://www.mathscipub.org

A NON-PROFIT CORPORATION

Typeset in LATEX

Copyright $\odot 2010$ by Mathematical Sciences Publishers 


\section{Algebra \& Number Theory}

Volume $4 \quad$ No. $8 \quad 2010$

On ramification filtrations and $p$-adic differential modules, I: the equal characteristic case

LIANG XIAO

Exponential generation and largeness for compact $p$-adic Lie groups

MICHAEL LARSEN

On the (non)rigidity of the Frobenius endomorphism over Gorenstein rings

HaIlong DaO, JinJia Li and Claudia Miller

A lower bound on the essential dimension of simple algebras

ALEXANDER S. MERKURJEV

On the minimal ramification problem for semiabelian groups

HERShy Kisilevsky, DANNy NEFTIN and JaCK SONN

Remarks on modular symbols for Maass wave forms

YURI I. MANIN 